\title{
Placental expression of ceruloplasmin in pregnancies complicated by severe preeclampsia
}

\author{
Seth Guller ${ }^{1}$, Catalin S Buhimschi ${ }^{1}$, Yula Y Ma ${ }^{1}$, Se Te J Huang ${ }^{1}$, Liubin Yang ${ }^{1}$, Edward Kuczynski ${ }^{1}$, \\ Eduardo Zambrano ${ }^{2}$, Charles J Lockwood ${ }^{1}$ and Irina A Buhimschi ${ }^{1}$
}

There is consensus that ischemia/reperfusion injury associated with preeclampsia (PE) promotes both placental damage and the release of factors leading to maternal endothelium dysfunction, a hallmark of this potentially life-threatening syndrome. These factors include plasminogen activator inhibitor-1 (PAI-1) and soluble fms-like tyrosine kinase-1 (sFlt-1). The goal of this study was to further characterize placental factors involved in the pathophysiology of PE. Thus, DNA microarray gene profiling was utilized to identify mRNA differentially regulated in placentas from women with severe PE compared to both preterm (PC) and term control (TC) groups. Microarray studies detected an upregulation of mRNA for ceruloplasmin, a copper-containing iron transport protein with antioxidant ferroxidase properties, in PE compared to PC and TC placentas, respectively. Quantitative real-time PCR confirmed these results by demonstrating significant increases in ceruloplasmin mRNA in PE vs PC and TC placentas. Supporting previous reports, the expression of sFlt-1 and PAI-1 were also upregulated in PE placentas. Immunohistochemistry localized ceruloplasmin to the intervillous space in PE and PC placentas, whereas stronger syncytial staining was noted in PE. Western blotting confirmed a significant increase in ceruloplasmin levels in placental tissue in PE compared to PC groups. PCR identified the presence of mRNA for ceruloplasmin in primary cultures of syncytiotrophoblasts, but not villous-derived fibroblasts, suggesting that syncytium is the site of ceruloplasmin synthesis in placenta. Hypoxic treatment $\left(1 \% \mathrm{O}_{2}\right)$ of syncytiotrophoblasts enhanced levels of ceruloplasmin mRNA approximately 25 -fold, a significantly greater upregulation than that noted for PAl-1 and sFlt-1, suggesting that enhanced ceruloplasmin expression is a sensitive marker of syncytial hypoxia. We suggest that syncytial ceruloplasmin and its associated ferroxidase activity, induced by the hypoxia accompanying severe PE, is important in an endogenous cellular program to mitigate the damaging effects of subsequent reperfusion injury at this site.

Laboratory Investigation (2008) 88, 1057-1067; doi:10.1038/labinvest.2008.74; published online 4 August 2008

KEYWORDS: placenta; preeclampsia; ceruloplasmin; hypoxia; oxygen radicals

The precise etiology of the maternal syndrome of preeclampsia $(\mathrm{PE})$ remains unknown. There is consensus that $\mathrm{PE}$ as well as many cases of idiopathic intrauterine growth restriction (IUGR), are strongly associated with failed conversion of maternal endometrial spiral arteries in the placental bed. ${ }^{1}$ This failure precludes physiological development of the low-resistance/high-capacitance uteroplacental circulation observed in uncomplicated pregnancies. ${ }^{2,3}$ Whether this is due to shallow trophoblast invasion and/or to decidual factors remains the subject of debate. ${ }^{1}$ It is suggested that hypoxia and/or reperfusion injury at the maternal-placental interface in PE leads to the release of soluble syncytiotrophoblast (SCT; ie the outer layer of the trophoblast in direct contact with maternal blood) factors including eicosanoids, ${ }^{4}$ peroxides, ${ }^{5,6}$ cytokines, ${ }^{7,8}$ SCT microparticles,, 90 soluble fms-like tyrosine kinase-1 (sFlt-1), ${ }^{11}$ endoglin, ${ }^{12}$ and plasminogen activator inhibitor-1 (PAI-1). ${ }^{13}$ These factors are postulated to contribute to placental damage and maternal endothelial dysfunction, hallmarks of PE, although their relative contributions in promoting clinical features of $\mathrm{PE}$ (ie maternal proteinuria and hypertension) remain to be determined.

The mechanism promoting the increased release of the abovementioned products by SCT in PE is largely unknown, although there is clear evidence of hypoxia-driven gene expression as well as oxidative damage in placentas from

\footnotetext{
'Department of Obstetrics/Gynecology and Reproductive Sciences, Yale University School of Medicine, New Haven, CT, USA and ${ }^{2}$ Department of Pathology, Yale University School of Medicine, New Haven, CT, USA

Correspondence: Dr S Guller, Department of Obstetrics/Gynecology and Reproductive Sciences, Yale University School of Medicine, 333 Cedar Street-339 FMB, PO Box 208063, New Haven, CT 06520-8063, USA.

E-mail: seth.guller@yale.edu

Received 10 March 2008; revised 10 June 2008; accepted 11 June 2008
} 
women with PE and idiopathic IUGR. ${ }^{14-16}$ Placental levels of the antiangiogenic factors, sFlt-1 and soluble endoglin, as well as the antifibrinolytic factor PAI-1, are all increased in pregnancies with PE. ${ }^{11-13}$ Conversely, levels of PAI-2, a major SCT protein, were lower in placentas from women with PE. ${ }^{13}$ Elevated expression of PAI-1 in SCT was specifically correlated with aberrantly high levels of intervillous fibrin and placental infarcts in patients with PE. ${ }^{13}$ The PAI-1, sFlt- 1 , and endoglin genes contain hypoxia response elements in their promoters and their expression in placental cultures is increased by hypoxia. ${ }^{17-19}$ It is known that the superoxide anion, the most common reactive oxygen species (ROS) can combine with nitric oxide to produce peroxynitrite anions which damage proteins. ${ }^{5}$ Increased placental nitrosylation of proteins and oxidative stress is a biochemical marker of $\mathrm{PE}^{14,15}$ In addition, ROS-associated lipid peroxidation is enhanced in placental villi from pregnancies complicated by PE. ${ }^{4,20}$ Placental adaptations to the presence of damaging oxygen radicals generated during hypoxia/reperfusion injury associated with PE may include activation of endogenous antioxidant enzymes including catalase ${ }^{21}$ and glutathione peroxidase. $^{22}$

The goal of the current study was to further characterize PE-associated placental pathophysiology. Gene profiling analysis of placental tissues was used to compare patterns of gene expression in placentas derived from women with $\mathrm{PE}$ compared to both preterm and term controls (PC and TC, respectively). This strategy revealed increased expression of ceruloplasmin, a copper-containing iron transport protein with ferroxidase activity, ${ }^{23,24}$ in PE compared to both PC and TC placentas. Validation of gene profiling results was accomplished by comparing placental expression of ceruloplasmin to that of sFlt-1 and PAI-1, which have been previously documented to be induced in PE. The likely cellular source of placental ceruloplasmin was then elucidated using placental tissue and primary cell cultures. Finally, modulation of ceruloplasmin expression by hypoxia was investigated, revealing a potential mechanism through which placental ceruloplasmin expression may be upregulated in pregnancies complicated by PE.

\section{MATERIALS AND METHODS}

\section{Patient Groups and Tissue Collection}

We studied placental samples from 32 women enrolled in the following groups:

(1) severe PE $(n=9)$; (2) PC $(n=16)$; and (3) TC $(n=7)$. Placentas were collected under protocols approved by the Human Investigation Committee of Yale University. Written informed consent was obtained from all participants, prior to enrollment. Gestational age was established based on menstrual date confirmed by sonographic examination prior to 20 weeks gestation. Characteristics of the study population are shown in Table 1.

Severe PE was defined based on the American College of Obstetricians and Gynecologists criteria: gestational age $>20$ weeks, systolic blood pressure of $160 \mathrm{~mm} \mathrm{Hg}$ or higher, or diastolic blood pressure of $110 \mathrm{~mm} \mathrm{Hg}$ or higher on two occasions at least $6 \mathrm{~h}$ apart, plus proteinuria of at least $5 \mathrm{~g}$ in a 24-hour urine specimen or a value of $3+$ or greater on dipstick testing of two random urine samples collected at least $4 \mathrm{~h}$ apart. ${ }^{25}$ As we have previously described, ${ }^{26}$ other elements of the diagnosis included IUGR $(<10$ th percentile for gestational age), cerebral or visual disturbances (headache, visual changes), epigastric or right upper-quadrant pain, pulmonary edema or cyanosis, oliguria (urinary output $<500 \mathrm{ml} / 24 \mathrm{~h}$ ), impaired liver function, and thrombocytopenia $(<100000$ cells $/ \mu \mathrm{l})$.

The PC group consisted of pregnancies with spontaneous preterm labor and/or preterm premature rupture of membranes with or without evidence of clinical or histological chorioamnionitis (CA). All patients in the PE and PC groups were administered a course of corticosteroids in compliance with previous clinical recommendations. ${ }^{27}$ Patients in the PC group had a clinically indicated amniocentesis to assess infection. Clinical CA was diagnosed by the presence of maternal fever (more than $37.8^{\circ} \mathrm{C}$ ), uterine tenderness, foul smelling amniotic fluid or visualization of pus at the time of the speculum examination, and maternal ( $>100$ beats per minute) or fetal ( $>160$ beats per minute) tachycardia. ${ }^{28,29}$ Histological examination of the placentas was performed by a perinatal pathologist (EZ), unaware of the clinical presentation or outcome. From each placenta, sections of chorionic plate, fetal membranes, and umbilical cord were examined for inflammation. Three histologic stages of CA (stage I: intervillositis; stage II: chorionic inflammation; and stage III: full-thickness inflammation of both chorion and amnion) were assigned based on a histologic grading system. ${ }^{30}$

Term placentas were obtained from uncomplicated pregnancies with appropriately grown singleton fetuses delivered at 37-40 weeks gestation by elective Cesarean section.

Following delivery, placentas were brought immediately to the laboratory and the decidua basalis layer from a central cotyledon, free of macroscopic pathology (ie fibrin deposition or infarcts), was dissected and discarded. Approximately $1 \mathrm{~g}$ of underlying villous tissue was collected and snap frozen in liquid nitrogen and maintained at $-80^{\circ} \mathrm{C}$ for use in gene array, quantitative real-time PCR (qRTPCR), and western blot studies. In addition, full thickness placental tissue specimens from both PE and PC groups $(n=5)$ were fixed in formalin for immunohistochemical analysis as described below. An additional five placentas from uncomplicated pregnancies, delivered at term by cesarean section, were used for the isolation of placental cell cultures.

\section{DNA Microarray Analysis}

RNA was extracted from snap-frozen placental samples from PE, PC, and TC groups $(n=3$ different placentas for each experimental group) using Trizol reagent (Sigma-Aldrich, St Louis, MO, USA). RNA was then purified using the RNeasy Mini Kit (Qiagen, Germantown, MD, USA), and 
Table 1 Characteristics of the study population at delivery $(n=32)$

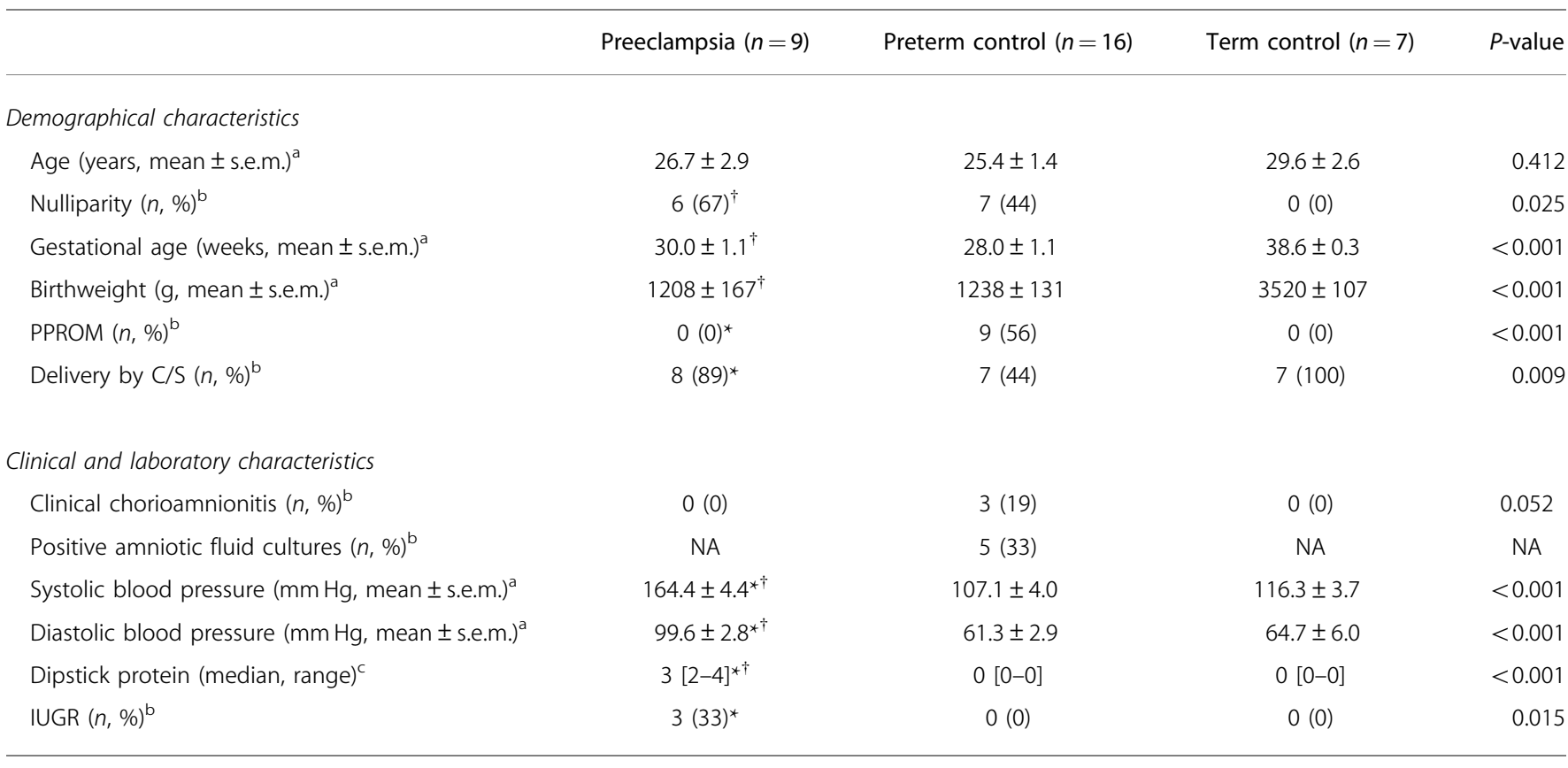

$C / S$, cesarean section; IUGR, intrauterine growth restriction; PPROM, preterm premature rupture of the membranes.

${ }^{\star} P<0.05$ preeclampsia vs preterm control; ${ }^{\dagger} P<0.05$ preeclampsia vs term control.

${ }^{\mathrm{a}}$ Data analyzed by one-way ANOVA.

bata analyzed by $\chi^{2}$ tests.

${ }^{\mathrm{C}}$ Data analyzed by Kruskal-Wallis ANOVA.

$20 \mu \mathrm{g}$ of purified RNA was submitted to the Keck Facility at Yale University School of Medicine for analysis of gene expression using the full human genome chip (HG_U133 Plus 2.0; Affymetrix, Santa Clara, CA, USA) containing approximately $54 \mathrm{~K}$ transcripts. Gene expression was analyzed using GCOS 1.4 GeneSpring (Affymetrix) and GX 7.3.1 (Agilant, San Jose, CA, USA) software. A twofold cut-off was used to establish an increase in gene expression as we have previously described. $^{31}$

\section{Quantitative Real-Time PCR}

RNA was extracted from placental tissue and $5 \mu \mathrm{g}$ was converted to cDNA. For qRTPCR, a standard curve was created between $500 \mathrm{pg}$ and $250 \mathrm{ng}$ of cDNA with a Roche Light Cycler (Roche, Indianapolis, IN, USA) by monitoring increasing fluorescence of PCR products during amplification as we have previously described. ${ }^{32}$ On establishing the standard curve, quantitation of the unknowns was determined with the Roche Light Cycler and adjusted to the expression of $18 \mathrm{~S}$ RNA from the corresponding unknowns. The primers used in this study are presented in Table 2 and were synthesized and gel purified at the Yale DNA Synthesis Laboratory, Critical Technologies. The identity of amplified products was confirmed by DNA sequencing. Alternatively, endpoint PCR was used to examine the expression of ceruloplasmin and $18 \mathrm{~S}$
RNA present in whole placental tissue or cultures of fibroblasts (FIBs) and SCTs using the primers described above.

\section{Immunohistochemistry}

Immunohistochemistry was used to localize ceruloplasmin in PE $(n=5)$ and PC placentas $(n=5)$ essentially using previously described techniques. ${ }^{32,33}$ Paraffin sections $(5 \mu \mathrm{m})$ of placental villous tissue were deparaffinized in xylene and rehydrated with graded ethanol to potassium-phosphatebuffered saline solution, $\mathrm{pH}$ 7.2. Following antigen retrieval with citrate buffer, the sections were pretreated with $1 \%$ hydrogen peroxide for $15 \mathrm{~min}$ and then incubated overnight at $4^{\circ} \mathrm{C}$ with primary antibody to ceruloplasmin (goat anti-human ceruloplasmin antibody, no. C0911 from Sigma-Aldrich) at a dilution of 1:100. For a negative control, serial sections were incubated with a 1:100 dilution of goat immunoglobulin G (IgG; Sigma-Aldrich, Cat. no. I5256). Both antibodies were used at a concentration of $10 \mu \mathrm{g} / \mathrm{ml}$. The slides were then incubated for $1 \mathrm{~h}$ at room temperature with biotinylated goat anti-rabbit IgG (Jackson Immunochemicals, West Grove, PA, USA; 1:600). Immunohistochemical staining was performed with avidin-biotin staining (Vectastain Elite ABC; Vector Laboratories, Burlingame, CA, USA) with 3,3'-diaminobenzidine/nickel sulfate as the chromogen solution. The tissue sections were dehydrated in graded ethanols, cleared, and mounted. 
Table 2 Primers for qRTPCR

\begin{tabular}{|c|c|c|c|}
\hline Primers & Forward $\left(5^{\prime}-3^{\prime}\right)$ & Reverse $\left(5^{\prime}-3^{\prime}\right)$ & Product (bp) \\
\hline Ceruloplasmin & CCCTGGAGAATGGATGCTCA & CTAACATGCTTCCCACGGATATT & 126 \\
\hline PAl-1 & TGCTGGTGAATGCCCTCTACT & CGGTCATTCCCAGGTTCTCTA & 399 \\
\hline sFlt-1 & TGTCACTGTTGCTAACTITC & AGAGGTTGGCATCAAAATG & 265 \\
\hline Cyclophilin B & CTCCTIGAGCTGTTGCAG & CACCACATGCTTGCCATCC & 325 \\
\hline
\end{tabular}

Visualization and photography was conducted with an IX71 inverted microscope (Olympus, Mellville, NY, USA). Representative micrographs are shown in Figure 3.

\section{Western Blotting}

Placental tissue extracts for immunoblotting were prepared by homogenization in buffer containing $50 \mathrm{mM}$ HEPES ( $\mathrm{pH}$ 7.5), $150 \mathrm{mM} \mathrm{NaCl}, 1 \mathrm{mM}$ EDTA, $1 \mathrm{mM}$ EGTA, $1 \mathrm{mM} \mathrm{NaF}$, $1 \%$ Triton X-100, 10\% glycerol, and additional protease and phosphatase inhibitors ( $1 \mathrm{mM}$ phenylmethylsulfonyl fluoride, $20 \mathrm{mM} \beta$-glycerophosphate, $8 \mathrm{mM}$ sodium pyrophosphate, $1 \mu \mathrm{g} / \mathrm{ml}$ leupeptin, $1 \mu \mathrm{g} / \mathrm{ml}$ pepstatin $\mathrm{A}$, and $1 \mu \mathrm{g} / \mathrm{ml}$ aprotinin (Roche Molecular Biochemicals, Indianapolis, IN, USA $))^{33}$ at a ratio of $0.1 \mathrm{~g}$ tissue $/ 1 \mathrm{ml}$ of extraction buffer. The lysates were centrifuged at 12000 r.p.m. for $15 \mathrm{~min}$ at $4{ }^{\circ} \mathrm{C}$. The supernatants were normalized for total protein concentration and samples were then boiled for $3 \mathrm{~min}$ in $2 \times$ SDS sample buffer with DTT. Approximately $100 \mu \mathrm{g}$ of protein was subjected to electrophoresis using $4-15 \%$ Tris- $\mathrm{HCl}$ polyacrylamide gradient gels. Following transfer, blots were incubated with primary antibody at $4{ }^{\circ} \mathrm{C}$ overnight at their respective dilutions: ceruloplasmin (sheep anti-human; Abcam, Cambridge, UK; no. ab8813, 1:400), cyclophilin B (rabbit anti-human; Abcam no. 3565, 1:2000). To detect ceruloplasmin and cyclophilin B, incubations with the appropriate secondary antibody conjugates and chemiluminescence substrate was carried out as we have previously described. ${ }^{33}$ Sheep serum (Sigma-Aldrich; no. S3772), used at a 1:400 dilution, replaced primary ceruloplasmin antibody as a control for western analysis. Western blot results were expressed as a ratio of ceruloplasmin/cyclophilin B obtained from densitometric analysis of autoradiographs using NIH ImageJ software (http://rsb.info.nih.gov/ij/).

\section{Cell Culture}

Primary cultures of cytotrophoblasts (CTs) were isolated from human term placentas $(n=5)$ and cultured using methodologies we have adopted ${ }^{33,34}$ from previously published procedure. ${ }^{35}$ Briefly, villous tissue was digested and separated on a continuous percoll gradient. To generate SCTs, CTs were maintained in medium with $10 \%$ fetal bovine serum (FBS; FBS medium) for $72 \mathrm{~h}$. Under these conditions
CTs spontaneously fuse to form SCTs. ${ }^{34,35}$ Placental villous FIBs were isolated based on our previous method, ${ }^{32,33}$ which is also a modification of an earlier methodology. ${ }^{36}$ For study, villous tissue was minced, digested, and dispersed cells were filtered through a $160 \mu \mathrm{m}$ stainless steel sieve. The filtrate was then centrifuged and resuspended in FBS medium. FIBs were subcultured and used between passages 3 and 10. Immunocytochemistry studies previously revealed that SCTs were positive for the trophoblast/epithelial cell marker cytokeratin 7 , but negative for vimentin (FIB marker) and $\alpha$-smooth muscle actin (myofibroblast/muscle cell marker). ${ }^{32,34}$ Cultured FIBs manifested a myofibroblast phenotype based on staining for vimentin and $\alpha$-smooth muscle actin, but not cytokeratin. $^{32,33}$

\section{Effects of Hypoxic Treatment on Gene Expression}

Cultures of SCTs from four different placentas were maintained for $24 \mathrm{~h}$ under normoxic conditions $\left(21 \% \mathrm{O}_{2}\right)$ in a standard incubator or under hypoxic conditions $\left(\begin{array}{ll}1 \% & \mathrm{O}_{2}\end{array}\right)$ using a controlled environment glovebox manufactured by Coy Laboratory Products (Grass Lake, MI, USA). The glove box design allows cell culture and treatment under a continuous hypoxic environment that is generated by a digitally regulated infusion of a preanalyzed gas mixture of $95 \% \mathrm{~N}_{2} /$ $5 \% \mathrm{CO}_{2}$. After incubation, RNA was isolated from hypoxic or normoxic cultures using Trizol. Following generation of cDNA, qRTPCR analysis of ceruloplasmin, PAI-1, and sFlt-1 expression was carried out as described above. Values were normalized to that of $18 \mathrm{~S}$ RNA and results from a single representative experiment are shown. In addition, cumulative results $(n=4)$, expressed as fold induction promoted by hypoxic treatment compared to normoxic levels, are also presented in Figure 6.

\section{Statistical Analysis}

We subjected all datasets to normality testing using the Kolmogorov-Smirnov method. Demographic, clinical, and laboratory characteristics are reported as a mean \pm s.e.m. Most comparisons between groups were performed using one-way analysis of variance (ANOVA). Kruskal-Wallis ANOVA was carried out for data that were not normally distributed and are presented as a mean with range. 
Proportions were compared with $\chi^{2}$-tests. Differences in placental gene and protein expression between groups measured by western blotting and by qRTPCR studies were analyzed by ANOVA and Student's $t$-test. In addition, ANOVA was used to analyze the effect of hypoxic treatment on expression of PAI-1, sFlt-1, and ceruloplasmin in cultures of SCTs. SigmaStat software was used for statistical analyses. A $P<0.05$ was considered significant in all studies.

\section{RESULTS}

\section{Characteristics of the Study Population}

The characteristics of the three groups in the study population are summarized in Table 1. Gestational age at delivery was significantly earlier in the PE $(n=9)$ group compared to TC $(n=7, P<0.001)$, but not significantly different than that of PC $(n=16)$. Three of the nine pregnancies from the PE group were also complicated by IUGR. Of the 16 pregnancies in the PC group, 5 showed evidence of clinical and histological CA and/or had positive amniotic fluid cultures.

\section{Expression of Ceruloplasmin mRNA in Placentas from PE, PC and TC Groups}

Gene array studies using three different placentas from each of the three study groups initially revealed that ceruloplasmin expression was enhanced 11- and 5-fold in PE vs PC $(P<0.01)$ and PE $v s$ TC $(P<0.01)$ groups, respectively. The gene array results are not yet published. qRTPCR was then carried out to verify changes in placental gene expression in the complete study population. We observed that levels of placental ceruloplasmin mRNA in the PE group, normalized to that of 18S RNA, was increased 32- and 4-fold that of the PC and TC groups, respectively $(P<0.001$ for both comparisons, Figure 1a). Similarly, when results were normalized using the housekeeping gene cyclophilin $\mathrm{B}$, ceruloplasmin expression in the PE group was upregulated approximately fivefold and threefold that of PC and TC groups $(P<0.05$ for both comparisons, Figure 1b). It is of note that in patients with isolated preterm IUGR, matched for gestational age with PE and PC groups, levels of placental ceruloplasmin expression were not increased (Figure 1c). This indicates that enhanced placental ceruloplasmin expression is associated with severe PE, not IUGR. In addition, in Figure 1c we also show that when the PE group is subdivided into patients with and without IUGR, and the PC group is subdivided into patients with (CA) and without (idiopathic preterm delivery) infection, ceruloplasmin levels in the PE only group was significantly increased compared to both PC groups. No other comparison between groups was significant. This demonstrated that placental ceruloplasmin levels are increased compared to gestational age-matched controls irrespective of infection. Thus, qRTPCR confirmed findings of the gene array studies and showed that levels of ceruloplasmin mRNA are increased in placentas from pregnancies with $\mathrm{PE}$ compared to PC and TC.
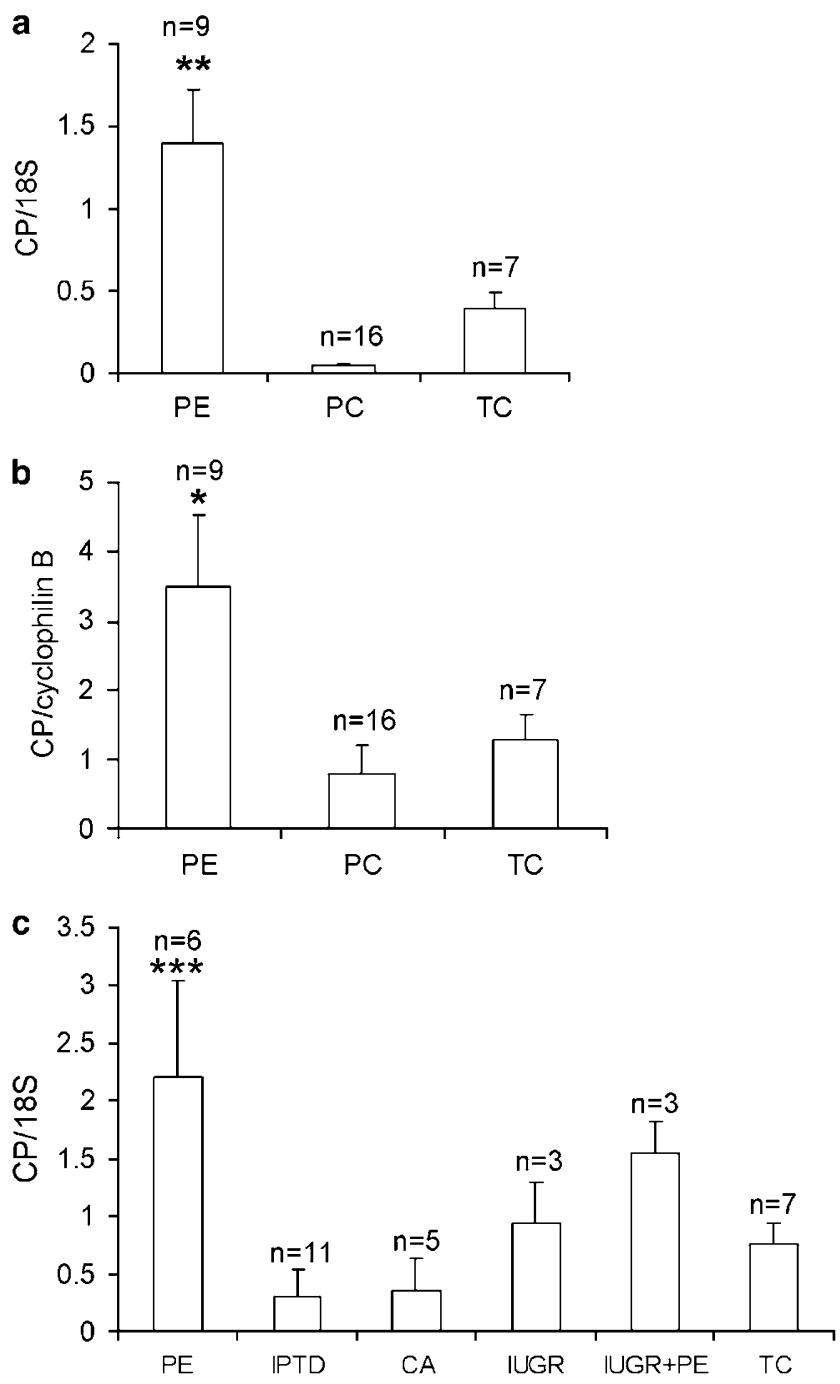

Figure 1 Quantitative real-time PCR (qRTPCR) analysis of placental ceruloplasmin expression. RNA was extracted from whole placental tissue from pregnancies with preeclampsia (PE), preterm control (PC), and term control (TC) groups and converted to CDNA. Levels of ceruloplasmin (CP) expression were then determined by qRTPCR and normalized to levels of 18S RNA (a) or cyclophilin B (b). In (c) the PE group was subdivided into patients without and with IUGR (PE and IUGR + PE, respectively), and the PC group was subdivided into patients without and with infection (idiopathic preterm delivery (IPTD) and chorioamnionitis (CA), respectively). Placentas from pregnancies with isolated IUGR were also analyzed. Results are expressed as a mean + s.e.m. from the indicated number of different placentas per group, and results were analyzed by ANOVA. ${ }^{\star} P<0.05$ vs PC and TC groups; ${ }^{* * P}<0.001$ vs PC and TC groups; ${ }^{* * * P}<0.05$ vs IPTD and CA groups.

As $\mathrm{PE}$ is known to be associated with increased placental levels of PAI- 1 and sFlt- $1,{ }^{11,13}$ we determined whether expression of these factors was induced in placentas from women with this condition. As shown in Figure 2a, qRTPCR revealed that PAI-1 levels in PE placentas were increased sevenfold that of PC $(P<0.001)$ and twofold that of TC $(P<0.01)$. Similarly, expression of sFlt-1 in PE placentas (Figure $2 \mathrm{~b})$ was increased 42 -fold that of PC $(P<0.001)$ and 

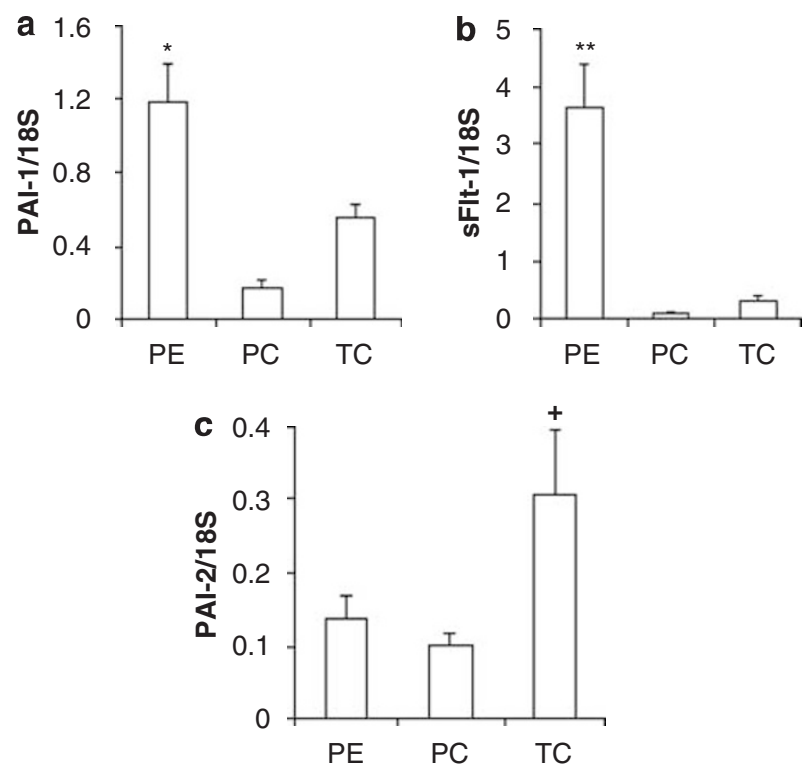

Figure 2 Analysis of placental PAI-1, sFlt-1, and PAI-2 expression in placentas by qRTPCR. Levels of placental PAI-1 (a), sFlt-1(b), and PAl-2 (c) gene expression in PE, PC, and TC groups were determined by qRTPCR and normalized to that of $18 \mathrm{~S}$ RNA. Nine, sixteen, and seven placentas were analyzed for all three genes in PE, PC, and TC groups, respectively. Results, expressed as a mean + s.e.m., were analyzed by ANOVA. ${ }^{*} P<0.01$ vs TC and $\mathrm{P}<0.001$ vs $\mathrm{PC} ;{ }^{* *} P<0.001$ vs $\mathrm{PC}$ and $\mathrm{TC}^{+}{ }^{+} P<0.01$ vs $\mathrm{PE}$ and $\mathrm{PC}$.

12 -fold that of TC $(P<0.001)$. Conversely, levels of PAI-2 mRNA in TC were approximately twofold higher than that found in PE $(P<0.05)$ and PC $(P<0.01)$ placentas (Figure $2 \mathrm{c})$. This result is consistent with the previous observation that $\mathrm{PE}$ was not associated with increased placental expression of PAI-2. ${ }^{13}$

\section{Placental Ceruloplasmin Protein Expression}

Immunohistochemistry was carried out to localize ceruloplasmin in placentas obtained in PE and PC groups $(n=5)$. Representative micrographs of each group are shown in Figure 3. Staining for ceruloplasmin was noted in the intervillous space in association with fibrin in both PE and PC groups, denoted by arrows in the low magnification micrographs in Figure 3a and b, respectively. In addition, syncytial staining for ceruloplasmin, denoted by arrowheads, was greater in the PE group under low (Figure 3a) and high magnification (Figure 3c), compared to PC (Figure $3 \mathrm{~b}$ and d). Staining within the villus core was primarily restricted to fetal capillaries in which ceruloplasmin was likely sequestered from fetal blood (Figure 3c). No staining was observed in the PE placenta when primary antibody was replaced with nonspecific goat IgG (Figure 3e).

Western blotting was employed to determine the level of ceruloplasmin protein expression in placental extracts from pregnancies with PE and PC $(n=6)$. We noted that ceruloplasmin was detected at a molecular weight of approximately $140 \mathrm{kDa}$, and a minor band was noted at $100 \mathrm{kDa}$
(Figure 4a, top blot). These results are consistent with molecular weights reported for placental forms of ceruloplasmin..$^{37,38}$ No signal was obtained when primary antibody was replaced with control sheep serum (not shown). Cyclophilin B, the housekeeping protein control, was detected at the predicted molecular weight of approximately $20 \mathrm{kDa}$ (Figure $4 \mathrm{a}$, bottom blot). ${ }^{39}$ Following normalization to cyclophilin B expression (Figure 4b), we observed that ceruloplasmin protein levels in the PE group were approximately threefold that found in the PC group $(P<0.01)$.

\section{Ceruloplasmin mRNA Expression in Placental Tissue and Cell Cultures}

RNA was then isolated from whole placental tissue as well as cultures of FIBs and SCTs obtained from three different placentas. Following generation of cDNA, levels of ceruloplasmin and 18S RNA expression were determined by conventional PCR. We noted that ceruloplasmin mRNA was expressed by term placental tissue and cultured SCTs, but not by FIBs (Figure 5, top panel). Levels of the 18S RNA housekeeping gene were similar in all samples (Figure 5, bottom panel). These results indicate that SCTs are the likely source of placental ceruloplasmin.

\section{Regulation of Syncytial Ceruloplasmin Expression by Hypoxic Treatment}

Cultures of SCTs were maintained for $24 \mathrm{~h}$ under normoxic or hypoxic conditions and levels of ceruloplasmin mRNA were determined following conversion to cDNA and analysis by qRTPCR. Expressions of PAI-1 and sFlt-1 were also examined because these factors have been demonstrated to increase in placenta under hypoxic conditions. ${ }^{17,18}$ Results were normalized to that of $18 \mathrm{~S}$ RNA. As shown in Figure 6a-c, the results from a single representative experiment indicated that hypoxic treatment enhanced expression of PAI-1, sFt-1, and ceruloplasmin approximately 1.6-, 6-, and 20-fold, respectively. Cumulative results from four independent experiments indicated that hypoxic treatment induced ceruloplasmin expression $23.7 \pm 3.2$-fold, significantly greater than the $1.7 \pm 0.2$-fold and $5.4 \pm 0.6$-fold induction in PAI- 1 and sFlt-1 levels, respectively $(P<0.001)$. This indicates that enhanced syncytial ceruloplasmin expression is a sensitive marker of syncytial hypoxia.

\section{DISCUSSION}

Ceruloplasmin was first described as a member of a coppercontaining oxidase family of enzymes. ${ }^{23}$ Despite this fact, ceruloplasmin is not involved in the transport or metabolism of copper. ${ }^{23}$ Decreased serum levels of ceruloplasmin were noted in patients with Wilson's disease, establishing the basis for a clinical test that is still used today. ${ }^{40}$ Early studies showed that iron accumulated in liver as well as tissues of pigs fed a copper-free diet, and administration of ceruloplasmin rapidly promoted the release of iron to the circulation. ${ }^{41,42}$ Using a dog liver perfusion system it was 

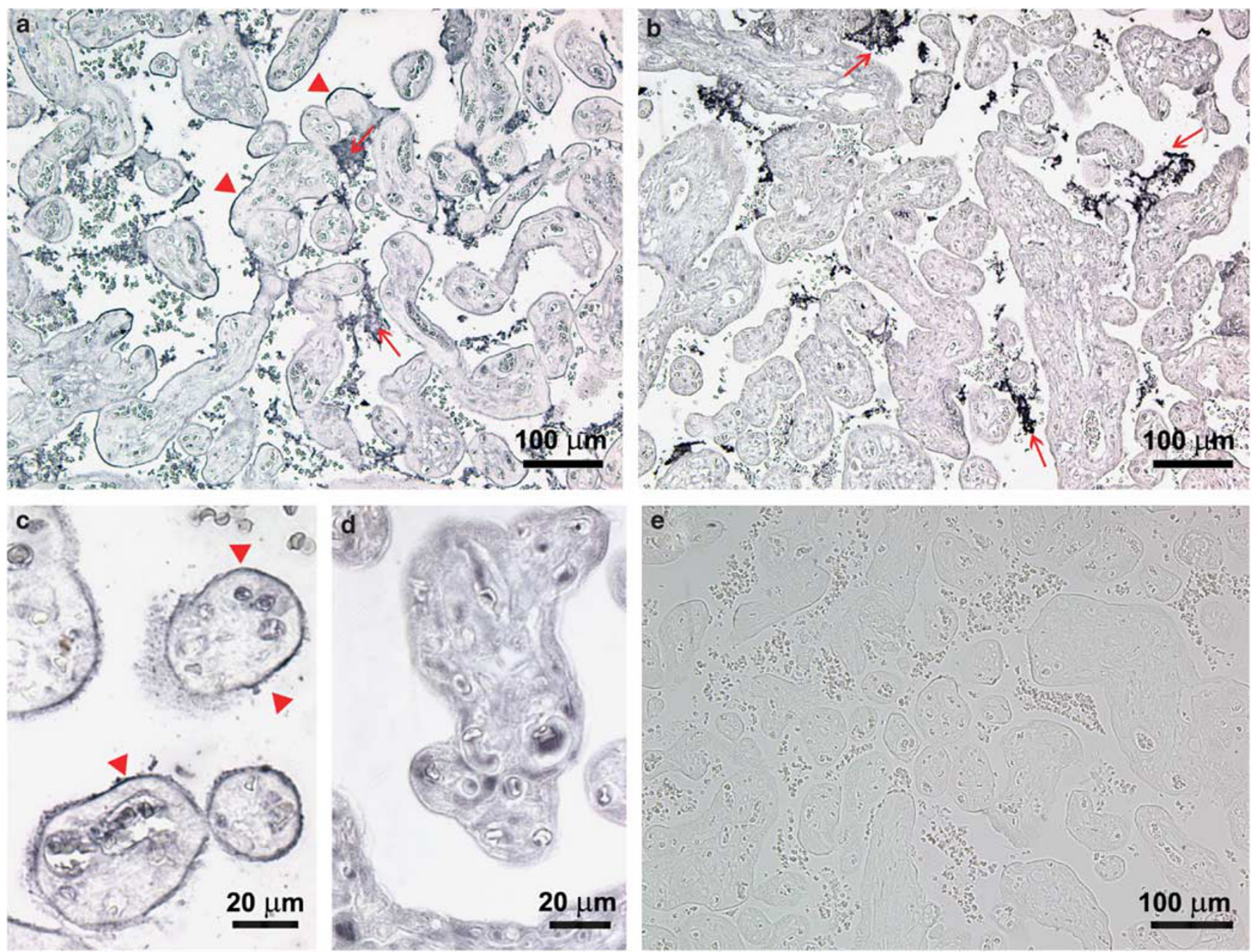

Figure 3 Immunohistochemical detection of ceruloplasmin in placenta. Immunohistochemistry was used to localize ceruloplasmin in villi of a placenta from PE (a, $\mathbf{c}$, and $\mathbf{e})$ and PC (b and $\mathbf{d})$ groups. These representative micrographs were from pregnancies delivered at approximately 31 weeks of gestation. Arrows indicate diaminobenzidine/nickel sulfate staining for ceruloplasmin in the intervillous space for both PE and PC placentas in (a) and (b), respectively. Conversely syncytial staining, denoted by arrowheads, was more intense in the PE placenta (a and $\mathbf{c}$ ). No staining was observed in the PE placenta when nonspecific goat IgG replaced the anticeruloplasmin antibody (e). (a), (b), and (e), $\times 200 ;(\mathbf{c})$ and $(\mathbf{d}), \times 640$.

demonstrated that ceruloplasmin treatment promoted a rapid release of iron by the liver which was incorporated into transferrin. ${ }^{43}$ Of note, ceruloplasmin was also demonstrated by the same group to be a ferroxidase. ${ }^{44}$ The ferroxidatic activity of ceruloplasmin is an important function of this enzyme because even trace amounts of iron can produce hydroxyl radicals through the Fenton reaction which can destroy cellular architecture. ${ }^{23,24}$ Ferroxidatic activity of ceruloplasmin is known to convert toxic ferrous iron to less toxic ferric iron, which reduces oxidative damage to lipids, proteins, and DNA. ${ }^{23,24}$

Ceruloplasmin is known to be an acute phase reactant, and its concentration in serum is upregulated during infection, inflammation, and tissue trauma, mediated by inflammatory cytokines. ${ }^{45,46}$ Its expression has also been shown to increase under hypoxic conditions. ${ }^{47}$ Although liver is its major source, ceruloplasmin has also been identified in several other tissues including spleen, lung, brain, testis, and placenta. ${ }^{48,49}$ More recent studies demonstrate that ceruloplasmin is synthesized as a glycophosphatidyl inositol-anchored protein generated by alternative splicing of exons 19 and 20 in Sertoli cells and astrocytes. ${ }^{50,51}$ In addition, a ceruloplasmin mRNA generated from alternative splicing of exon 18 has also been detected in extrahepatic cells. ${ }^{52}$ In the current study we used primers that amplified sequences from 340 to 465 in the ceruloplasmin gene located in exons 2 and 3. Therefore, our methodology would detect all relevant ceruloplasmin mRNA species. The circulating form of ceruloplasmin is a protein with 1046 amino acids with a molecular weight of approximately $132 \mathrm{kDa}^{23}$ Studies using antibody to circulating ceruloplasmin detected a protein homolog in BeWo choriocarcinoma cells at molecular weights of 140 and $110 \mathrm{kDa}^{37,38}$ Our western blot studies revealed a predominant species at $140 \mathrm{kDa}$ in placental tissue. As our 

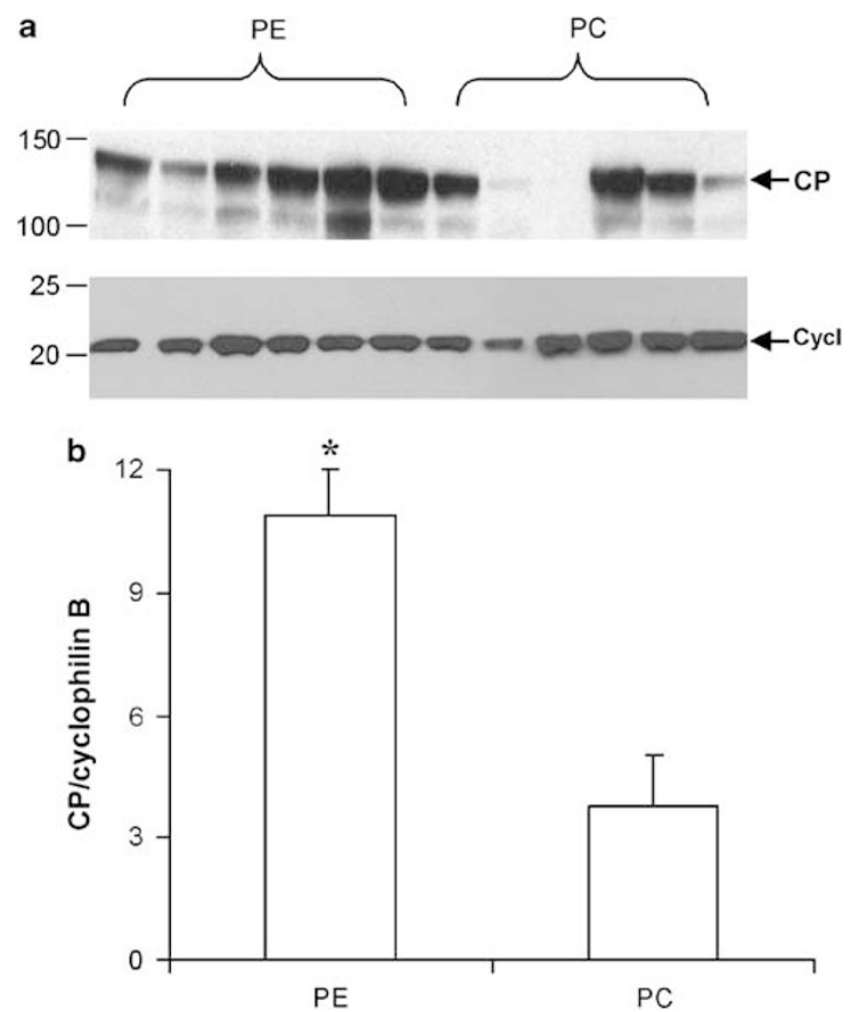

Figure 4 Detection of placental ceruloplasmin expression by western blotting. Western blot analysis of whole placental tissue extracts was carried out to assess the relative levels of ceruloplasmin in PE and PC groups ( $n=6$ different placentas per group). Ceruloplasmin (CP) was detected at a molecular weight of approximately $140 \mathrm{kDa}$ (top blot, a), cyclophilin B (Cycl) served as a housekeeping protein control (bottom blot, a). Quantitation of ceruloplasmin protein levels was performed by densitometry following normalization to cyclophilin B expression and is presented in (b). Results are expressed as a mean (cerulolplasmin/ cyclophilin ratio $\times 10)+$ s.e. The values for the $P E$ and $P C$ groups were $10.9 \pm 2.7$ and $3.8 \pm 3.4$, respectively. ${ }^{\star} P<0.01$ vs $P C$ group by Student's $t$-test.

present work and prior studies ${ }^{37,38}$ detected ceruloplasmin in placental cell extracts and tissue using an antibody directed against the circulating form, this suggests that the same epitope is present in placental tissue and in circulating forms released by the liver.

Our results specifically indicated that placental levels of ceruloplasmin are increased in pregnancies with severe PE. This conclusion is based on several lines of experimental evidence. First, gene array analysis and qRTPCR revealed increased ceruloplasmin expression in placentas from pregnancies with PE compared to both PC and TC groups. This indicated that levels of placental ceruloplasmin mRNA are elevated in pregnancies with PE whether or not gestational age at delivery is controlled for. It is of note, that ceruloplasmin mRNA was not altered in placentas from pregnancies with CA compared to gestational age-matched PCs without clinical and histological evidence of infection. This demonstrated that infection per se does not regulate placental

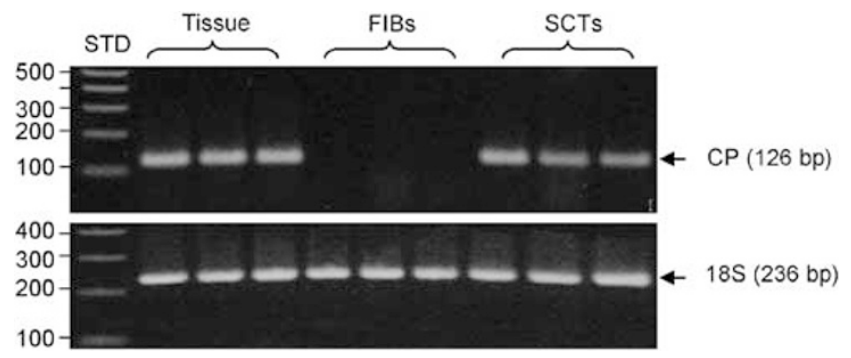

Figure 5 PCR analysis of ceruloplasmin expression in tissue and cells isolated from term placentas. RNA was extracted from whole placental tissue, or cultures of syncytiotrophoblasts (SCTs) and fibroblasts (FIBs) obtained from three different placentas. Following conversion to CDNA, endpoint PCR was carried out to detect ceruloplasmin ( $C P$, top panel) and 18S RNA (bottom panel). The size of the amplified product is indicated at the right of the figure.

ceruloplasmin expression. Although it is acknowledged that there is no true 'control' group for patients with preterm PE, our results clearly demonstrate that placental ceruloplasmin expression is clearly upregulated in the PE group vs patients matched for gestational age. In addition, our qRTPCR results also demonstrate that ceruloplasmin levels were not elevated in patients with isolated IUGR. Thus, although the etiology of PE and IUGR are both suggested to result from failed conversion of maternal spiral arteries early in pregnancy, ${ }^{1}$ this indicated that enhanced placental ceruloplasmin expression accompanies severe PE, not IUGR.

It was noted by qRTPCR that levels of the housekeeping gene cyclophilin $\mathrm{B}$ were not statistically different in the PE, PC, and TC groups (not shown). Levels of 18S RNA were comparable in $\mathrm{PE}$ and TC, but levels of $18 \mathrm{~S}$ RNA in the PE group were approximately one-half that of PC $(P<0.05)$. Potential variation in placental housekeeping gene expression in PE led to our decision to use two genes to normalize qRTPCR results. Thus, it is likely that the induction of placental ceruloplasmin gene expression in patients with $\mathrm{PE}$ compared to PC may be closer to the 5-fold increase found when cyclophilin B was used to normalize results, rather than the 32 -fold increase when 18S RNA was used. Gene array results, which are not standardized by housekeeping genes, revealed an 11-fold induction in the PE group vs PC.

Our western blot results revealed increased levels of ceruloplasmin protein in the PE group of placentas compared to the PC group, although this difference was not as large as that noted for changes in mRNA expression. There are several potential explanations for this observed difference in relative levels of placental ceruloplasmin protein and mRNA expression. The most likely one is that tissue extracts used for western blotting contained placental ceruloplasmin as well as circulating forms of this protein present in maternal and fetal blood. The levels of ceruloplasmin in maternal and cord blood were found to range from approximately 200 to $900 \mu \mathrm{g} / \mathrm{ml}$ and 60 to $120 \mu \mathrm{g} / \mathrm{ml}$, respectively. ${ }^{53-55}$ In fact, immunohistochemistry did reveal ceruloplasmin staining in 
a

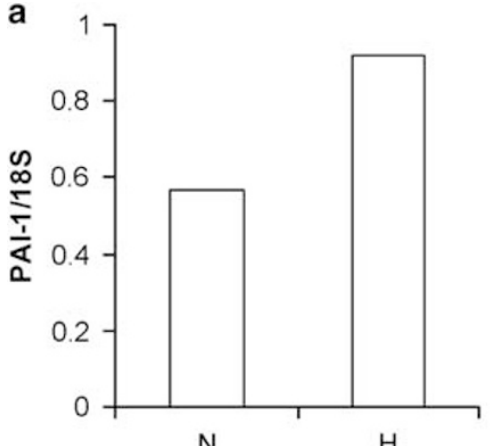

N b

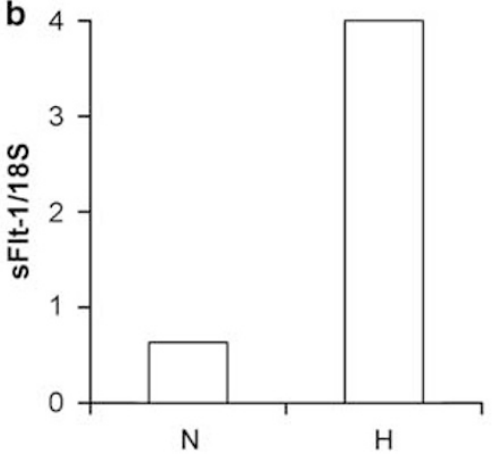

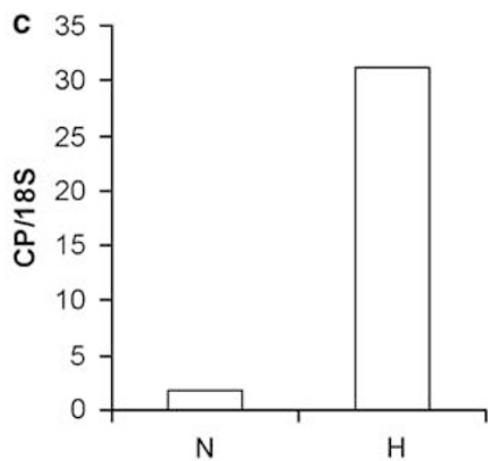

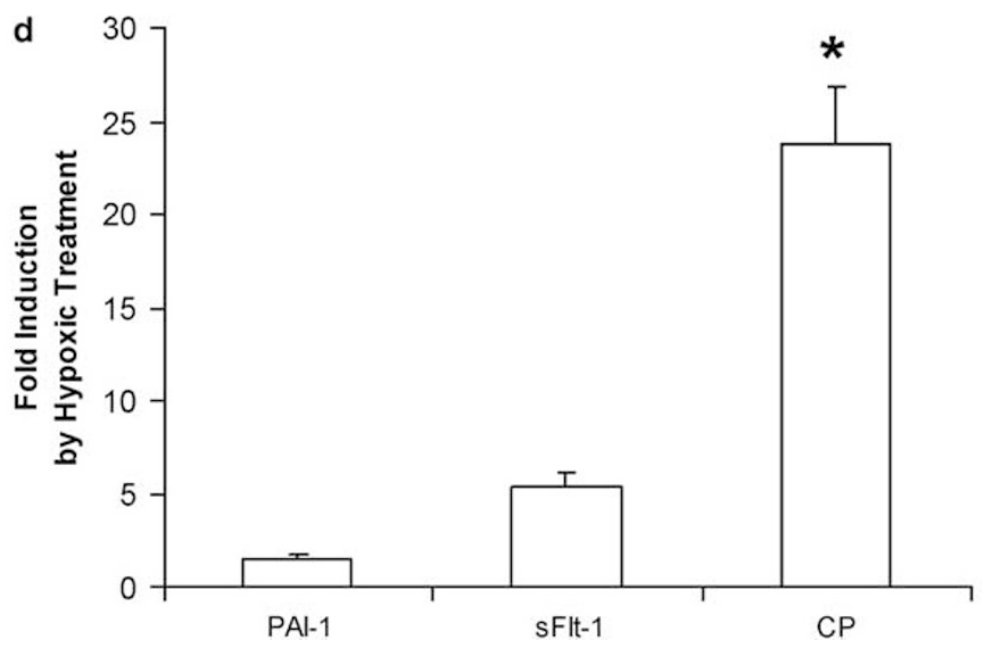

Figure 6 Induction of ceruloplasmin expression in SCTs by hypoxic treatment. Primary cultures of SCTs were maintained for $24 \mathrm{~h}$ under normoxic (N) or hypoxic $(\mathrm{H})$ conditions and levels of PAI-1 (a), sFlt-1 (b), and ceruloplasmin (CP, c) were determined by qRTPCR and normalized to level of $18 \mathrm{~S}$ RNA. Panels a to $c$ depict results from a single representative experiment. Cumulative results from four independent experiments, expressed as fold induction by hypoxic treatment (mean + s.e.m.), are shown in $(\mathbf{d}) .{ }^{*} P<0.001$ vs PAl-1 and sFlt-1 by ANOVA.

the intervillous space of both PE and PC groups, most likely reflecting the presence of maternal protein at this site. Thus, western blot results may have underestimated the relative difference in placental ceruloplasmin levels between PE and PC groups due to the presence of maternal ceruloplasmin in tissue extracts. It is also possible that changes in protein or mRNA stability are responsible for the somewhat discordant results of ceruloplasmin protein and mRNA expression.

Following the establishment of increased ceruloplasmin expression in placentas from pregnancies with $\mathrm{PE}$, we then sought to determine its potential cellular source. Immunohistochemistry revealed more prominent syncytial staining in the PE group compared to PC. However, because SCTs are in direct contact with maternal blood, syncytial staining detected by immunohistochemistry could be due to maternal ceruloplasmin bound to the surface of chorionic villi. To determine which cell types in placenta may synthesize ceruloplasmin, we then isolated RNA from whole tissue and primary cultures of SCTs and FIBs, and examined ceruloplasmin expression by PCR. Our results indicate that SCTs express ceruloplasmin mRNA and are the likely source of placental ceruloplasmin. It is of note, that $\mathrm{PE}$ was associated with a $60 \%$ increase in ceruloplasmin levels in maternal serum in one study ${ }^{53}$ and a $16 \%$ increase in another. ${ }^{55}$ It has not been determined whether placental ceruloplasmin is secreted, thus its contribution to the PE-associated increase of this protein in maternal serum is not known.

Given our finding that placental ceruloplasmin levels are increased in PE, the question arises as to which factors may lead to its enhanced expression in these pregnancies. One possibility is that placental hypoxia associated with $\mathrm{PE}^{1,2}$ increases placental ceruloplasmin expression as has been noted for macrophages and monocytes. ${ }^{47}$ Our results using primary cultures of SCTs show that ceruloplasmin expression is exquisitely sensitive to hypoxic treatment. In fact, the approximate 25 -fold induction in ceruloplasmin expression was significantly greater than the effect on expression of PAI-1 and sFlt-1. It is relevant that placental levels of both PAI-1 and sFlt-1 are increased in pregnancies with $\mathrm{PE},{ }^{11,13}$ and were demonstrated to be induced in trophoblast under hypoxic conditions. ${ }^{18,56}$ Thus, our results suggest that ceruloplasmin may be a sensitive marker of syncytial hypoxia in pregnancies with PE. We did not detect secreted ceruloplasmin in conditioned media from cultures of SCTs by western blotting. 
This suggests that levels of ceruloplasmin were most likely below the sensitivity of the assay, or possibly, the epitope detected in ceruloplasmin released by the liver was absent in ceruloplasmin secreted by SCTs in vitro.

Several recent studies have used gene array technology to identify alterations in placental gene expression associated with PE. ${ }^{16,57-59}$ Only one specifically identified ceruloplasmin to be increased in expression in placentas with PE compared to gestational-age matched controls, ${ }^{16}$ but this finding was not verified by qRTPCR, nor was the analysis of cell-type specific expression and regulation of ceruloplasmin documented as in the present report. There are several possible explanations why other studies did not report changes in placental ceruloplasmin expression in association with PE. Firstly, we only included placentas derived from women with severe preterm PE or HELLP syndrome. Secondly, placentas were obtained from term deliveries in one study. ${ }^{59}$ Although corticosteroids were administered to all patients in PE and PC groups in our study, this issue is not addressed in another report. ${ }^{57}$ In addition, racial and/or ethnic differences of patient study groups may also exist, tissue preparation and RNA extraction protocols vary, and gene array studies cast a wide net and not all differences may be reported.

It is interesting to speculate as to the potential role of elevated placental ceruloplasmin in PE. The disorder is characterized by increased placental expression of ROS, lipid peroxidation, and damage to villous architecture. ${ }^{4,5,15,20}$ It has been suggested that increased placental expression of the antioxidant enzymes glutathione peroxidase and catalase may serve as an adaptive/protective mechanism to limit oxidative damage in PE. ${ }^{21,22}$ Our findings suggest that increased levels of placental ceruloplasmin in PE may result in enhanced ferroxidatic activity in this tissue, thereby oxidizing excess ferrous iron to the less toxic ferric form. We conclude that syncytial ceruloplasmin, induced by hypoxia associated with severe PE, may be important in an endogenous cellular program to mitigate the damaging effects of subsequent reperfusion injury at this site.

\section{ACKNOWLEDGEMENTS}

We thank Luisa Coraluzzi for procuring term placentas for the isolation of cultures of syncytiotrophoblasts and fibroblasts. This work was supported in part by Grant HD33909 from the NIH (SG).

1. Redman CW, Sargent IL. Latest advances in understanding preeclampsia. Science 2005;308:1592-1594.

2. Kaufmann P, Black S, Huppertz B. Endovascular trophoblast invasion: implications for the pathogenesis of intrauterine growth retardation and preeclampsia. Biol Reprod 2003;69:1-7.

3. Stepan H, Faber R, Dornhofer N, et al. New insights into the biology of preeclampsia. Biol Reprod 2006;74:772-776.

4. Walsh SW, Vaughan JE, Wang $\mathrm{Y}$, et al. Placental isoprostane is significantly increased in preeclampsia. FASEB J 2000;14:1289-1296.

5. Myatt $L$, Cui $X$. Oxidative stress in the placenta. Histochem Cell Biol 2004;122:369-382.

6. Sikkema JM, van Rijn BB, Franx A, et al. Placental superoxide is increased in pre-eclampsia. Placenta 2001;22:304-308.
7. Benyo DF, Smarason A, Redman CW, et al. Expression of inflammatory cytokines in placentas from women with preeclampsia. J Clin Endocrinol Metab 2001;86:2505-2512.

8. Conrad KP, Miles TM, Benyo DF. Circulating levels of immunoreactive cytokines in women with preeclampsia. Am J Reprod Immunol 1998;40:102-111.

9. Goswami D, Tannetta DS, Magee LA, et al. Excess syncytiotrophoblast microparticle shedding is a feature of early-onset pre-eclampsia, but not normotensive intrauterine growth restriction. Placenta 2006;27: 56-61.

10. Knight $M$, Redman CW, Linton EA, et al. Shedding of syncytiotrophoblast microvilli into the maternal circulation in preeclamptic pregnancies. Br J Obstet Gynaecol 1998;105:632-640.

11. Maynard SE, Min JY, Merchan J, et al. Excess placental soluble fms-like tyrosine kinase 1 (sFlt1) may contribute to endothelial dysfunction, hypertension, and proteinuria in preeclampsia. J Clin Invest 2003;111:649-658.

12. Venkatesha $S$, Toporsian $M$, Lam $C$, et al. Soluble endoglin contributes to the pathogenesis of preeclampsia. Nat Med 2006;12:642-649.

13. Estelles A, Gilabert J, Keeton $M$, et al. Altered expression of plasminogen activator inhibitor type 1 in placentas from pregnant women with preeclampsia and/or intrauterine fetal growth retardation. Blood 1994;84:143-150.

14. Many A, Hubel CA, Fisher SJ, et al. Invasive cytotrophoblasts manifest evidence of oxidative stress in preeclampsia. Am J Pathol 2000;156:321-331.

15. Myatt $\mathrm{L}$, Rosenfield RB, Eis AL, et al. Nitrotyrosine residues in placenta. Evidence of peroxynitrite formation and action. Hypertension 1996;28:488-493.

16. Soleymanlou N, Jurisica I, Nevo O, et al. Molecular evidence of placental hypoxia in preeclampsia. J Clin Endocrinol Metab 2005;90:4299-4308.

17. Meade ES, Ma YY, Guller S. Role of hypoxia-inducible transcription factors 1alpha and 2alpha in the regulation of plasminogen activator inhibitor-1 expression in a human trophoblast cell line. Placenta 2007;28:1012-1019.

18. Nagamatsu T, Fujii $T$, Kusumi $M$, et al. Cytotrophoblasts up-regulate soluble fms-like tyrosine kinase-1 expression under reduced oxygen: an implication for the placental vascular development and the pathophysiology of preeclampsia. Endocrinology 2004;145:4838-4845.

19. Yinon $Y$, Nevo O, Xu J, et al. Severe intrauterine growth restriction pregnancies have increased placental endoglin levels: hypoxic regulation via transforming growth factor- 3. Am J Pathol 2008;172:77-85.

20. Cester N, Staffolani R, Rabini RA, et al. Pregnancy induced hypertension: a role for peroxidation in microvillus plasma membranes. Mol Cell Biochem 1994;131:151-155.

21. Wang Y, Walsh SW. Antioxidant activities and mRNA expression of superoxide dismutase, catalase, and glutathione peroxidase in normal and preeclamptic placentas. J Soc Gynecol Investig 1996;3:179-184.

22. Knapen MF, Peters WH, Mulder TP, et al. Glutathione and glutathionerelated enzymes in decidua and placenta of controls and women with pre-eclampsia. Placenta 1999;20:541-546.

23. Hellman NE, Gitlin JD. Ceruloplasmin metabolism and function. Annu Rev Nutr 2002;22:439-458.

24. Patel BN, Dunn RJ, Jeong SY, et al. Ceruloplasmin regulates iron levels in the CNS and prevents free radical injury. J Neurosci 2002;22: 6578-6586.

25. ACOG practice bulletin. Diagnosis and management of preeclampsia and eclampsia. Number 33, January 2002 Obstet Gynecol 2002;99:159-167.

26. Buhimschi CS, Norwitz ER, Funai E, et al. Urinary angiogenic factors cluster hypertensive disorders and identify women with severe preeclampsia. Am J Obstet Gynecol 2005;192:734-741.

27. Effect of corticosteroids for fetal maturation on perinatal outcomes. $\mathrm{NIH}$ consensus development panel on the effect of corticosteroids for fetal maturation on perinatal outcomes. JAMA 1995;273:413-418.

28. Newton ER. Preterm labor, preterm premature rupture of membranes, and chorioamnionitis. Clin Perinatol 2005;32:571-600.

29. Pettker CM, Buhimschi IA, Magloire LK, et al. Value of placental microbial evaluation in diagnosing intra-amniotic infection. Obstet Gynecol 2007;109:739-749.

30. Salafia CM, Weigl C, Silberman L. The prevalence and distribution of acute placental inflammation in uncomplicated term pregnancies. Obstet Gynecol 1989;73:383-389. 
31. Huang SJ, Schatz F, Masch R, et al. Regulation of chemokine production in response to pro-inflammatory cytokines in first trimeste decidual cells. J Reprod Immunol 2006;72:60-73.

32. Ma Y, Krikun G, Abrahams VM, et al. Cell type-specific expression and function of toll-like receptors 2 and 4 in human placenta: implications in fetal infection. Placenta 2007;28:1024-1031.

33. Lee $M J$, Wang $\mathrm{Z}$, Yee $\mathrm{H}$, et al. Expression and regulation of glucocorticoid receptor in human placental villous fibroblasts. Endocrinology 2005;146:4619-4626.

34. Guller S, LaCroix NC, Kirkun G, et al. Steroid regulation of oncofetal fibronectin expression in human cytotrophoblasts. J Steroid Biochem Mol Biol 1993;46:1-10.

35. Kliman HJ, Nestler JE, Sermasi E, et al. Purification, characterization, and in vitro differentiation of cytotrophoblasts from human term placentae. Endocrinology 1986;118:1567-1582.

36. Fant ME, Nanu L. Human placental endothelin: expression of endothelin-1 mRNA by human placental fibroblasts in culture. Mol Cel Endocrinol 1995;109:119-123.

37. Danzeisen R, Fosset C, Chariana Z, et al. Placental ceruloplasmin homolog is regulated by iron and copper and is implicated in iron metabolism. Am J Physiol Cell Physiol 2002;282:C472-C478.

38. Danzeisen R, Ponnambalam S, Lea RG, et al. The effect of ceruloplasmin on iron release from placental (BeWo) cells; evidence for an endogenous Cu oxidase. Placenta 2000;21:805-812.

39. Price ER, Zydowsky LD, Jin MJ, et al. Human cyclophilin B: a second cyclophilin gene encodes a peptidyl-prolyl isomerase with a signal sequence. Proc Natl Acad Sci USA 1991;88:1903-1907.

40. Scheinberg IH, Gitlin D. Deficiency of ceruloplasmin in patients with hepatolenticular degeneration (Wilson's disease). Science 1952;116:484-485.

41. Lee GR, Nacht S, Lukens JN, et al. Iron metabolism in copper-deficient swine. J Clin Invest 1968;47:2058-2069.

42. Roeser HP, Lee GR, Nacht $\mathrm{S}$, et al. The role of ceruloplasmin in iron metabolism. J Clin Invest 1970;49:2408-2417.

43. Osaki S, Johnson DA, Frieden $\mathrm{E}$. The mobilization of iron from the perfused mammalian liver by a serum copper enzyme, ferroxidase I. J Biol Chem 1971;246:3018-3023.

44. Osaki S, Johnson DA, Frieden E. The possible significance of the ferrous oxidase activity of ceruloplasmin in normal human serum. J Biol Chem 1966;241:2746-2751.

45. Barber EF, Cousins RJ. Interleukin-1-stimulated induction of ceruloplasmin synthesis in normal and copper-deficient rats. J Nutr 1988; 118:375-381.
46. Gitlin JD. Transcriptional regulation of ceruloplasmin gene expression during inflammation. J Biol Chem 1988;263:6281-6287.

47. Sarkar J, Seshadri V, Tripoulas NA, et al. Role of ceruloplasmin in macrophage iron efflux during hypoxia. J Biol Chem 2003;278: 44018-44024.

48. Aldred AR, Grimes A, Schreiber G, et al. Rat ceruloplasmin. Molecular cloning and gene expression in liver, choroid plexus, yolk sac, placenta, and testis. J Biol Chem 1987;262:2875-2878.

49. Fleming RE, Gitlin JD. Primary structure of rat ceruloplasmin and analysis of tissue-specific gene expression during development. J Biol Chem 1990;265:7701-7707.

50. Fortna RR, Watson HA, Nyquist SE. Glycosyl phosphatidylinositolanchored ceruloplasmin is expressed by rat Sertoli cells and is concentrated in detergent-insoluble membrane fractions. Biol Reprod 1999;61:1042-1049.

51. Patel BN, David S. A novel glycosylphosphatidylinositol-anchored form of ceruloplasmin is expressed by mammalian astrocytes. J Biol Chem 1997;272:20185-20190.

52. Yang FM, Friedrichs WE, Cupples RL, et al. Human ceruloplasmin. Tissue-specific expression of transcripts produced by alternative splicing. J Biol Chem 1990;265:10780-10785.

53. Engin-Ustun $\mathrm{Y}$, Ustun $\mathrm{Y}$, Kamaci $\mathrm{M}$, et al. Maternal serum ceruloplasmin in preeclampsia. Int J Gynaecol Obstet 2005;89:51-52.

54. Galinier A, Periquet B, Lambert W, et al. Reference range for micronutrients and nutritional marker proteins in cord blood of neonates appropriated for gestational ages. Early Hum Dev 2005;81:583-593.

55. Serdar Z, Gur E, Develioglu O. Serum iron and copper status and oxidative stress in severe and mild preeclampsia. Cell Biochem Funct 2006;24:209-215.

56. Fitzpatrick TE, Graham CH. Stimulation of plasminogen activator inhibitor-1 expression in immortalized human trophoblast cells cultured under low levels of oxygen. Exp Cell Res 1998;245: 155-162.

57. Hansson SR, Chen Y, Brodszki J, et al. Gene expression profiling of human placentas from preeclamptic and normotensive pregnancies. Mol Hum Reprod 2006;12:169-179.

58. Reimer T, Koczan D, Gerber B, et al. Microarray analysis of differentially expressed genes in placental tissue of pre-eclampsia: up-regulation of obesity-related genes. Mol Hum Reprod 2002;8:674-680.

59. Zhou R, Zhu Q, Wang Y, et al. Genomewide oligonucleotide microarray analysis on placentae of pre-eclamptic pregnancies. Gynecol Obstet Invest 2006;62:108-114. 